\title{
Relationship between Depression and Treatment Satisfaction among Patients with Type 2 Diabetes
}

Jon Bassett ${ }^{1}$, Alan Adelman ${ }^{2}$, Robert Gabbay, ${ }^{3,4 *}$ and Raquel M Aňel-Tiangco ${ }^{4}$

${ }^{1}$ University of Nebraska Medical Center and Ehrling Bergquist at Offutt AFB, USA

${ }^{2}$ Penn State Hershey Medical Group Fishburn Road, Hershey, PA, USA

${ }^{3}$ Penn State Hershey Diabetes Institute, Hershey, PA, USA

${ }^{4}$ Division of Endocrinology, Diabetes and Metabolism, Penn State College of Medicine, PA, USA

\begin{abstract}
Background: Depression has been shown to adversely affect glycemic control. The purpose of this study is to examine the association between depression and treatment satisfaction in patients with diabetes.

Materials and methods: Baseline data was collected on 545 patients with poorly controlled type 2 diabetes enrolled in a study that examined the effectiveness of diabetes nurse case managers. Depression was measured using the Center for Epidemiologic Studies Depression (CES-D) questionnaire, and treatment satisfaction, using the Diabetes Treatment Satisfaction Questionnaire (DTSQ).

Results: The majority of participants $(59 \%)$ were female, with a high percentage $(41 \%)$ of Hispanic/Latino participants with a mean $\mathrm{HbA} 1 \mathrm{C}$ of $8.4 \%$. The prevalence of depression in this population was $35.6 \%$. High CES-D scores were associated with elevated levels of HbA1C and LDL cholesterol $(p<0.001)$. The relationship between depression and treatment satisfaction was significant $(p<0.001)$, indicating that as depression increases, treatment satisfaction decreases.

Discussion: We identified a significant relationship between depression and treatment satisfaction in this group of poorly controlled type 2 diabetes patients. Although causation cannot be determined, it is possible that patients who are depressed are less likely to be satisfied with their treatment. This could lead to decreased patient adherence, ultimately resulting in poor glycemic control.
\end{abstract}

Keywords: Depression; Treatment satisfaction; Type 2 diabetes

\section{Introduction}

Depression is prevalent in the diabetes population. A systematic meta-analysis by Anderson et al. indicated that the odds of comorbid depression doubled for patients with diabetes compared to those without it. The prevalence of depression was also significantly higher among women than in men with diabetes [1]. A more recent metaanalysis reported the prevalence of depression in patients with type 2 diabetes to be $17.6 \%$, compared with $9.8 \%$ among control subjects [2].

This apparent increased risk of comorbid depression amongst the diabetes population can have many worrisome outcomes. Depression has been associated with hyperglycemia in patients with both type 1 and type 2 diabetes in a meta-analysis by Lustman et al. [3]. Comorbid depression has also been associated with increased risk of diabetic retinopathy, nephropathy, neuropathy, macrovascular complications, and sexual dysfunction [4].

Although an association exists between depression and hyperglycemia, causality is less clear. Studies have shown that reducing depression leads to improvement in hemoglobin A1C levels in diabetes patients [3]. Likewise, improving HbAlC levels with aggressive hypoglycemic pharmacotherapy has been shown to improve depression $[5,6]$. Thus, an improvement in glycemic control or depression consequently leads to an improvement in the other. However, the reason for this association remains unclear. A meta-analysis has shown that patients who are depressed are 3 times more likely to be nonadherent with treatment compared to their non-depressed counterparts [7]. It has become increasingly important to assess patient satisfaction as it relates to adherence, and it is unclear what factors influence treatment satisfaction. Amongst patients with diabetes, lower treatment satisfaction resulted in greater difficulties with adherence to treatment
[8]. The purpose of this study was to explore the relationship between depression, diabetes treatment satisfaction, and health outcomes.

\section{Materials and Methods}

\section{Population}

Baseline data on 545 participants was collected as part of a randomized National Institute of Diabetes and Digestive and Kidney Diseases (NIDDK) funded clinical trial known as the DYNAMIC study (Diabetes nurse case management and motivational interviewing for change) [9]. Patients eligible for the study were identified based on billing data, searching the Penn State Diabetes Registry and subsequent chart review from nine Penn State Hershey Medical Center primary care offices and three Reading Hospital-affiliated primary care clinics that serve a predominantly Hispanic population [10]. Inclusion criteria consisted of patients' age $>18$ years with poorly controlled type 2 diabetes or its comorbidities as indicated by one of the following: HbA1C $>8.5$, LDL $>130$, or blood pressure $(\mathrm{BP})>140 / 90$. Patients who were pregnant, unable to communicate in English or Spanish, or were in nursing homes were excluded from the study.

*Corresponding author: Robert Gabbay, Penn State Hershey Diabetes Institute, 500 University Drive, H044, Hershey, PA 17033, USA, Tel: 717-531-3592; Fax: 717531-5726; E-mail: rgabbay@psu.edu

Received July 09, 2012; Accepted August 19, 2012; Published August 22, 2012

Citation: Bassett J, Adelman A, Gabbay R, Aňel-Tiangco RM (2012) Relationship between Depression and Treatment Satisfaction among Patients with Type 2 Diabetes. J Diabetes Metab 3: 210. doi:10.4172/2155-6156.1000210

Copyright: ( 2012 Bassett J, et al. This is an open-access article distributed unde the terms of the Creative Commons Attribution License, which permits unrestricted use, distribution, and reproduction in any medium, provided the original author and source are credited. 


\section{Data collection}

Patients completed the Center for Epidemiologic Studies Depression (CES-D) questionnaire. The CES-D is a standardized, self report questionnaire with a maximum score of 60 points. Higher scores indicate greater psychological distress. A score $\geq 16$ is indicative of clinically significant psychological distress. In the general population it would be expected that $\sim 20 \%$ of people would record a score $\geq 16$ [11].

The 8-item Diabetes Treatment Satisfaction Questionnaire (DTSQ) was used to determine patient treatment satisfaction. It is the most widely used and psychometrically evaluated measure of patient satisfaction with diabetes treatment $[12,13]$. Four items of the questionnaire specifically assess satisfaction, convenience, flexibility, and understanding of treatment. Two additional questions address the patients' willingness to continue with treatment as well as the likelihood that they would recommend such treatment to others. The final two questions of the survey tool address the patients' awareness of hyperand hypoglycemia. There is a maximum score of 36 points, with higher scores indicating higher satisfaction with treatment [13].

Clinical data (HbA1C, LDL, BP) were collected through the Penn State Diabetes Registry [10]. Chart abstractors were used to ensure that all the information was up to date.

\section{Analysis}

Minitab version 15.1.2 (Minitab, Inc., State College, Pennsylvania) was used for the analysis. We initially performed univariate analyses on all demographic variables (age, gender, race, education level, income, and marital status), health outcome variables (BMI, HbA1C, LDL, BP), and survey data (CES-D and DTSQ scores). Next, Pearson's correlation coefficient was calculated, searching for relationships between demographic variables, health outcomes, and survey data. Pearson's correlation statistics were then calculated comparing CES-D and DTSQ in greater detail. To compare categorical variables with health outcomes and survey results a one-way ANOVA was used. Depression was then looked at as a categorical variable, with CES-D scores $\geq 16$ used to indicate depression. A two-sample $\mathrm{T}$ test was used to observe differences in health outcomes and survey data between patients who were considered depressed and those who did not meet the criteria. Finally, a stepwise regression model was used to assess the statistically significant relationships between CES-D scores and demographic variables, health outcomes, and other survey data, with an alpha-toenter of 0.15 and an alpha-to-remove of 0.15 .

\section{Results}

The baseline demographics and clinical measures data on a total of 545 patients who participated in the study are shown in table 1 . The majority of the patients were female and middle-aged. Most were Caucasian, although there was also a large number of Hispanic/Latino patients $(n=212,41 \%)$. Most of them reported making less than $\$ 35,000$ annually $\$ 3,000$ monthly.

One-third (33\%) of the patients had a baseline HbA1C measurement of $\leq 7 \%$, indicating good glycemic control. In addition, $42 \%$ of patients had $\mathrm{LDL} \leq 100 \mathrm{mg} / \mathrm{dL}, 42 \%$ had a systolic $\mathrm{BP} \leq 130 \mathrm{mmHg}$, and $70 \%$ had a diastolic $\mathrm{BP} \leq 80 \mathrm{mmHg}$, all of which are target levels for diabetes patients. The study was designed specifically to look at poorly controlled patients. As such, only 10 patients $(3.9 \%)$ were well-controlled in all three parameters of $\mathrm{HbA1C}$, LDL, and blood pressure.

HbA1C was lowest in Caucasians $(8.1 \%, \mathrm{p}<0.01)$ and in patients who had some form of college degree $(8.1 \%, \mathrm{p}<0.01)$. Conversely,

\begin{tabular}{|c|c|c|}
\hline Characteristic & Results & $\mathbf{N}^{*}$ \\
\hline \multicolumn{3}{|l|}{ Gender } \\
\hline Female & $59 \%$ & 320 \\
\hline Male & $41 \%$ & 225 \\
\hline Age in years, mean (SD) & $58(11.41)$ & 531 \\
\hline \multicolumn{3}{|l|}{ Race } \\
\hline Caucasian & $49 \%$ & 254 \\
\hline Hispanic/Latino & $41 \%$ & 212 \\
\hline Black/African American & $7 \%$ & 37 \\
\hline Other & $3 \%$ & 19 \\
\hline \multicolumn{3}{|l|}{ Highest Education } \\
\hline No High School Degree & $34 \%$ & 168 \\
\hline High School Degree or Equivalent & $31 \%$ & 156 \\
\hline College and Beyond & $35 \%$ & 173 \\
\hline \multicolumn{3}{|l|}{ Annual Household Income } \\
\hline Less than $\$ 35,000 / \$ 36,000$ & $71 \%$ & 332 \\
\hline$\$ 35,000 / \$ 36,000-\$ 65,000 / \$ 66,000$ & $15 \%$ & 70 \\
\hline Greater than $\$ 65,000 / \$ 66,000$ & $14 \%$ & 68 \\
\hline \multicolumn{3}{|l|}{ Marital Status } \\
\hline Married & $52 \%$ & 268 \\
\hline Single & $20 \%$ & 103 \\
\hline Other & $28 \%$ & 148 \\
\hline \multicolumn{3}{|l|}{ Clinical Measures } \\
\hline BMI, mean (SD) & $34.4(8.25)$ & 490 \\
\hline $\mathrm{A} 1 \mathrm{C}$, mean (SD) & $8.4(2.15)$ & 485 \\
\hline LDL, mean (SD) & $114(39.41)$ & 497 \\
\hline Systolic BP, mean (SD) & $137(19.33)$ & 296 \\
\hline Diastolic BP, mean (SD) & $77(11.73)$ & 296 \\
\hline A1C control $(\leq 7)$ & $33 \%$ & 159 \\
\hline LDL control ( $\leq 100$ mg/dL) & $42 \%$ & 210 \\
\hline Systolic BP control $(\leq 130 \mathrm{mmHg})$ & $42 \%$ & 125 \\
\hline Diastolic BP control $(\leq 80 \mathrm{mmHg})$ & $70 \%$ & 208 \\
\hline Overall Control (A1C, LDL, BP) & $3.9 \%$ & 10 \\
\hline \multicolumn{3}{|l|}{ Survey Data } \\
\hline CES-D score, mean (SD) & $14.8(15.02)$ & 523 \\
\hline DTSQ score, mean (SD) & $26.6(8.23)$ & 538 \\
\hline
\end{tabular}

*Total of 545 patients. Missing data were excluded from analysis.

Table 1: Baseline Demographics, Outcome Data, and Survey Results.

HbA1c was highest in Hispanic patients $(8.84 \%, \mathrm{p}<0.01)$ and in those with no high school degree $(8.85 \%, \mathrm{p}<0.01)$. CES-D scores were significantly lower (indicating lower levels of distress) in Caucasians, in patients with some educational degree, in those who reported an annual income $>\$ 65,000 / \$ 66,000$, and in those who were married. The patient groups that were more likely to be satisfied with their treatment included the Hispanic patients, those without a high school degree, and those with an annual household income of $<\$ 35,000 / \$ 36,000$.

A total of 186 patients (35.6\%) scored $\geq 16$ on the CES-D questionnaire, indicating clinically significant psychological distress. As shown in table 2, CES-D was found to be significantly correlated to some of the clinical outcome variables. Specifically, as CES-D scores increased, HbAlc, LDL, and diastolic BP increased, and treatment satisfaction decreased.

An analysis of the correlation between CES-D and DTSQ by demographic factors is shown in table 3. Overall, as CES-D scores increased, treatment satisfaction decreased. This relationship was stronger in men than women, in Caucasian than in Hispanic/Latino participants, and was strongest in male Caucasian participants. 


\begin{tabular}{|l|l|}
\hline Outcomes & CES-D \\
\hline A1C & $0.158 \ddagger$ \\
\hline LDL & $0.153 \ddagger$ \\
\hline SBP & 0.002 \\
\hline DBP & $0.139^{\star}$ \\
\hline DTSQ & $-0.201 \ddagger$ \\
\hline
\end{tabular}

${ }^{*} \mathrm{p} \leq 0.05$

$\neq \mathrm{p} \leq 0.001$

Table 2: Pearson's correlation coefficients of CES-D and clinical outcome variables.

\begin{tabular}{|l|l|}
\hline Variables & Pearson's Correlation \\
\hline Female & $-0.146^{\star}$ \\
\hline Males & $-0.286 \ddagger$ \\
\hline Hispanic/Latino & $-0.139^{*}$ \\
\hline Caucasian & $-0.325 \ddagger$ \\
\hline Female Hispanic/Latino & $-0.107^{*}$ \\
\hline Male Hispanic/Latino & $-0.239^{\star}$ \\
\hline Female Caucasian & $-0.297 \ddagger$ \\
\hline Male Caucasian & $-0.333 \ddagger$ \\
\hline $\begin{array}{l}\text { Results after adjusting for the effects of age, gender, } \\
\text { BMI, income, race, marital status, and education }\end{array}$ & $-0.255 \ddagger$ \\
\hline
\end{tabular}

${ }^{*} p<0.05$

$+\mathrm{p}<0.01$

$\ddagger p<0.001$

Table 3: Correlation between CES-D and DTSQ by demographic factors.

\section{Discussion}

The purpose of this study was to examine the association of depression and treatment satisfaction in a population of individuals with type 2 diabetes. The prevalence of depression amongst this group of poorly controlled type 2 diabetes patients (mean $\mathrm{HbA} 1 \mathrm{C}=8.4 \%$ was $35.6 \%$ using a CES-D score $\geq 16$ as a cutoff for depression). Roughly $20 \%$ of the general population typically would record a score $\geq 16$. Therefore, the prevalence of depression is nearly double in this population, which is consistent with the literature regarding the high prevalence of depression among persons with diabetes [2].

There was a clear relationship between depression and treatment satisfaction. As scores on the CES-D rose, indicating higher levels of distress, treatment satisfaction scores decreased. Similar results have been described among depressed patients with coronary artery disease [14]. It is unknown whether poor treatment satisfaction leads to greater distress or whether greater distress negatively impacts the patient's view of his or her diabetes treatment satisfaction. Further trials aimed at examining the impact of depression on treatment satisfaction will need to be performed to explore this relationship and potential point of intervention.

While this study has much strength including a diverse population, validated surveys, and inclusion of clinical parameters, this study has several limitations. First, because of its cross-sectional study design, causation cannot be determined. Although the demonstrated relationship between depression and diabetes treatment satisfaction has face validity, the current study design cannot determine causation. Second, this study may be limited because it focused on patients who are uncontrolled, with the inclusion criteria being that they are uncontrolled in HbA1C levels, LDL levels, and/or blood pressure. This may not be representative of the general diabetes population. As the literature suggests, poorer glycemic control can be a risk factor for depression and depression can also be a risk factor for developing diabetes $[1,2]$. By focusing on a specific population of diabetes patients who are not well-controlled, the results from our study could be skewed and cannot be generalized to the population of individuals with type 2 diabetes. Third, we do not know whether the subjects were diagnosed as being clinically depressed and whether they were receiving medication and/or counseling. This would be very important for a longitudinal study that examined prospectively the effect of treatment on depression and diabetes treatment satisfaction. And finally, treatment adherence was not measured in this study. Again this will be an important factor to include in any prospective study.

In conclusion, there is still a need for further research to assess the relationship between depression and treatment satisfaction among diabetes patients. If improving treatment satisfaction leads to improvement in depression scores, then treatment satisfaction can be targeted as an area of improvement. It would also be interesting to see whether addressing either depression or treatment satisfaction would affect patient adherence and ultimately, if this will improve clinical outcomes.

\section{Acknowledgments}

Support was provided by the National Institute of Diabetes and Digestive and Kidney Diseases grant R18-DK067495

\section{References}

1. Anderson RJ, Freedland KE, Clouse RE, Lustman PJ (2001) The prevalence of comorbid depression in adults with diabetes: a meta-analysis. Diabetes Care 24: 1069-1078.

2. Ali S, Stone MA, Peters JL, Davies MJ, Khunti K (2006) The prevalence of co-morbid depression in adults with Type 2 diabetes: a systematic review and meta-analysis. Diabet Med 23: 1165-1173.

3. Lustman PJ, Anderson RJ, Freedland KE, de Groot M, Carney RM, et al. (2000) Depression and poor glycemic control: a meta-analytic review of the literature. Diabetes Care 23: 934-942.

4. de Groot M, Anderson R, Freedland KE, Clouse RE, Lustman PJ (2001) Association of depression and diabetes complications: a meta-analysis. Psychosom Med 63: 619-630.

5. Mazze RS, Lucido D, Shamoon H (1984) Psychological and social correlates of glycemic control. Diabetes Care 7: 360-366.

6. Testa MA, Simonson DC (1998) Health economic benefits and quality of life during improved glycemic control in patients with type 2 diabetes mellitus: a randomized, controlled, double-blind trial. JAMA 280: 1490-1496.

7. DiMatteo MR, Lepper HS, Croghan TW (2000) Depression is a risk factor for noncompliance with medical treatment: Meta-analysis of the effects of anxiety and depression on patient adherence. Arch Intern Med 160: 2101-2107.

8. Biderman A, Noff E, Harris SB, Friedman N, Levy A (2009) Treatment satisfaction of diabetic patients: what are the contributing factors? Fam Pract 26: 102-108.

9. Stuckey HL, Dellasega C, Graber NJ, Mauger DT, Lendel I, et al. (2009) Diabetes nurse case management and motivational interviewing for change (DYNAMIC): study design and baseline characteristics in the Chronic Care Model for type 2 diabetes. Contemp Clin Trials 30: 366-374.

10. Khan L, Mincemoyer S, Gabbay RA (2009) Diabetes registries: where we are and where are we headed? Diabetes Technol Ther 11: 255-262.

11. Radloff LS (1977) The CES-D scale: a self report depression scale for research in the general population. Appl Psychol Meas 1: 385-401.

12. Witthaus E, Stewart J, Bradley C (2001) Treatment satisfaction and psychological well-being with insulin glargine compared with NPH in patients with Type 1 diabetes. Diabet Med 18: 619-625.

13. Bradley C (1994) Handbook of Psychology and Diabetes: A Guide to Psychological Measurement in Diabetes Research and Practice. Harwood Academic Publishers, Chur, Switzerland.

14. Spertus JA, McDonell M, Woodman CL, Finn SD (2000) Association between depression and worse disease-specific functional status in outpatients with coronary artery disease. Am Heart J 140: 105-110. 